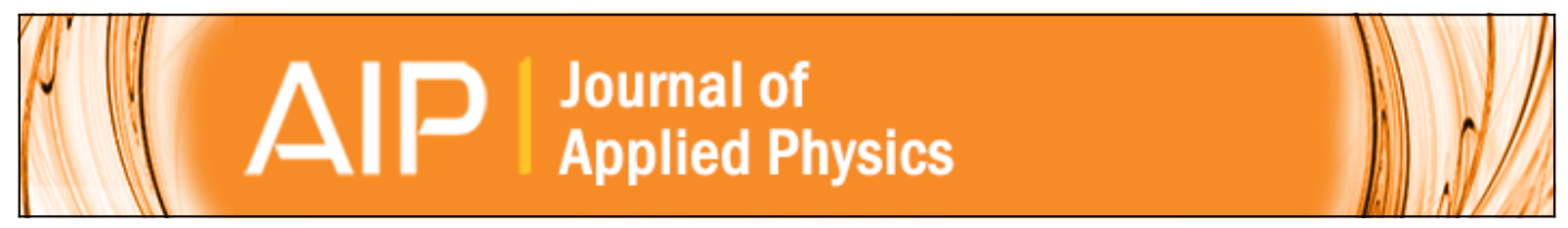

\title{
Three-dimensional fluid simulation of a plasma display panel cell
}

H. C. Kim, M. S. Hur, S. S. Yang, S. W. Shin, and J. K. Lee

Citation: Journal of Applied Physics 91, 9513 (2002); doi: 10.1063/1.1479758

View online: http://dx.doi.org/10.1063/1.1479758

View Table of Contents: http://scitation.aip.org/content/aip/journal/jap/91/12?ver=pdfcov

Published by the AIP Publishing

\section{Articles you may be interested in}

Mechanism of high luminous efficacy in plasma display panel with high secondary electron emission coefficient cathode material analyzed through three-dimensional fluid model simulation

J. Appl. Phys. 110, 043303 (2011); 10.1063/1.3626455

Simulation of striation in large-gap coplanar plasma display panels

Phys. Plasmas 17, 033510 (2010); 10.1063/1.3361198

Three-dimensional spatiotemporal behaviors of light emission from discharge plasma of alternating current plasma display panels

Appl. Phys. Lett. 92, 221506 (2008); 10.1063/1.2938074

Numerical study on microdischarges in plasma display panels with arbitrary cell geometry

J. Appl. Phys. 98, 053302 (2005); 10.1063/1.2001145

Three-dimensional self-consistent radiation transport model for the fluid simulation of plasma display panel cell J. Appl. Phys. 93, 9516 (2003); 10.1063/1.1577399

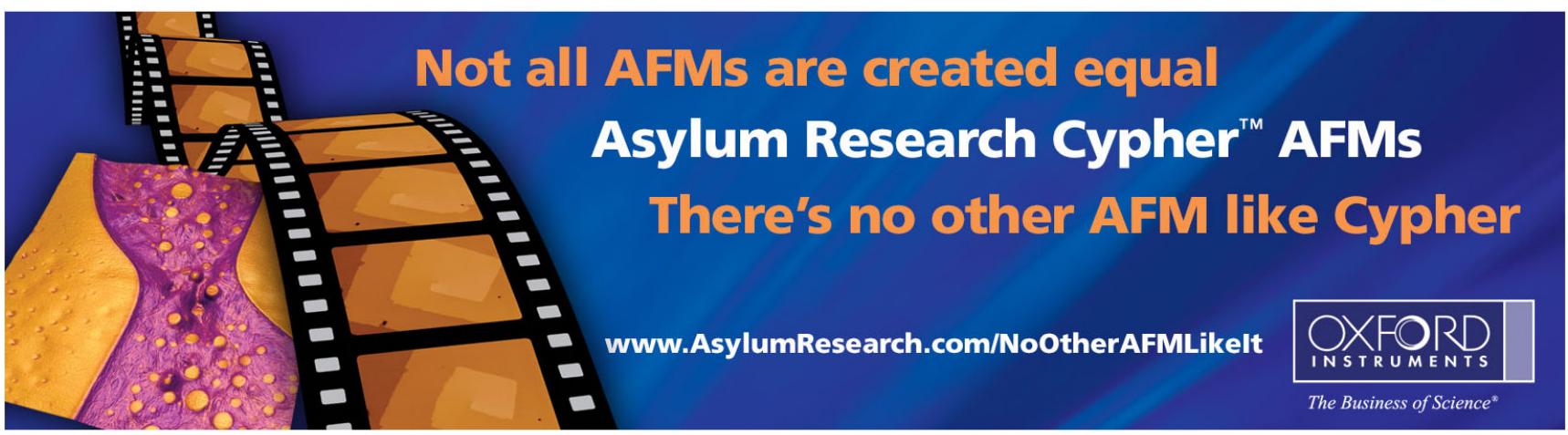




\title{
Three-dimensional fluid simulation of a plasma display panel cell
}

\author{
H. C. Kim, M. S. Hur, S. S. Yang, S. W. Shin, and J. K. Lee ${ }^{\text {a) }}$ \\ Department of Electronic and Electrical Engineering, Pohang University of Science and Technology, \\ Pohang 790-784, South Korea
}

(Received 19 September 2001; accepted for publication 28 March 2002)

\begin{abstract}
In order to understand the discharge characteristics in an alternating current plasma display panel (ac PDP) and optimize it further, a three-dimensional fluid code (FL3P) has been developed. Using this simulator, various three-dimensional features of discharges are investigated in the sustain mode of PDP. First, the striations of wall charge are observed at both the anode and cathode side. Second, the local efficiency is obtained as a function of position. It is mainly divided into the anode region and the cathode region and highest near the anode center. Finally, the effects of various three-dimensional parameters are studied. As one of the examples showing the effect of electrode shaping, the discharge characteristics of a T-shaped electrode cell are compared with those of a conventional cell. The phosphor on barrier ribs contributes to over $44 \%$ of the total luminance, but barrier ribs themselves do not play an important role in the overall discharge efficiency. Address electrode width is not always proportional to the size of the discharge because of the wall loss of the particles to barrier ribs. (C) 2002 American Institute of Physics. [DOI: 10.1063/1.1479758]
\end{abstract}

\section{INTRODUCTION}

Plasma display panels (PDPs) are one of the most promising technologies for wall hanging high definition color television. ${ }^{1}$ However, there remain important issues to be solved, such as improvement of luminous efficiency, reducing power consumption, and increasing the lifetime. Optimizing the properties of PDP can be aided by understanding the microdischarge physics inside a PDP cell.

The light emission of PDP is based on high-pressure glow discharge whose typical dimensions are in a few hundred $\mu \mathrm{m}$ range and which forms the basic picture element of the display. In the monochrome display, the visible light from the discharge is directly used, while the color display panel uses the ultraviolet (UV) photons that are converted into the visible photons by red, green, and blue phosphors. The UV photons are generated from the discharge of neon, helium, and xenon gas mixture. Sometimes argon or other rare gases are also mixed.

The PDP cells are confined to a volume of submillimeter gap size. Direct experimental access and diagnostics of the discharge are limited because of the small system size. Numerical simulation can therefore be a useful tool to provide detailed information on the microdischarge characteristics in a PDP cell. There have been many one- and two-dimensional numerical models of PDP discharges over the last a few years. ${ }^{2-7}$ One-dimensional models are sufficient for simulating matrix-type $\mathrm{PDP}^{2-4}$ while the study of coplanar-type PDP requires at least two-dimensional modeling. ${ }^{5-7}$ Because of its low speed, three-dimensional simulations are not common, though three-dimensional variations of the cell structure are widely performed in experiments. Examples are PDPs with T-shaped electrodes ${ }^{8}$ and delta tri-color arrangement cell structures with meander barrier ribs, ${ }^{9}$ which have

\footnotetext{
a)Electronic mail: jkl@postech.ac.kr
}

higher luminous efficiency. Therefore, three-dimensional simulations are considered important for further optimization and proposing new designs of PDP cells, but there are not many three-dimensional models. ${ }^{10,11}$

In this article, we report on a three-dimensional fluid code (FL3P) for PDP. Previously, we have successfully developed a two-dimensional code for PDP (FL2P) ${ }^{12}$ and have reported on certain aspects of optimization. ${ }^{13}$ The FL3P is a generalization of the FL2P that utilizes the local field approximation and the drift-diffusion model. The semi-implicit coupling of Poisson's equation and the continuity equation makes it possible to increase the simulation time step above that of the dielectric relaxation time. Consequently, the simulation speed is greatly enhanced. Taking advantage of its fast computation speed, we focus here on the simulation of the sustain mode rather than that of the firing mode that is mainly simulated in other three-dimensional simulations.

We describe the physical model and numerical method in Sec. II. Section III shows the discharge characteristics in the sustain mode and the three-dimensional features, especially, the effects of the three-dimensional parameters such as electrode shape, barrier ribs, and width of address electrode. We also suggest the future direction of a cell design for the high efficiency. Section IV summarizes our work.

\section{DESCRIPTION OF THE MODEL}

\section{A. Physical model}

Our model consists of the following set of coupled equations:

$$
\begin{aligned}
& \frac{\partial n_{i}}{\partial t}+\nabla \cdot \boldsymbol{\Gamma}_{i}=S_{i}, \\
& \boldsymbol{\Gamma}_{i}=-D_{i} \nabla n_{i}+\operatorname{sgn}\left(q_{i}\right) \mu_{i} n_{i} \mathbf{E},
\end{aligned}
$$




$$
\nabla \cdot(\epsilon \mathbf{E})=\sum_{i} q_{i} n_{i}
$$

where $i$ stands for the electron, ions (i.e., $\mathrm{Xe}^{+}$and $\mathrm{Ne}^{+}$), or excited species (i.e., $\mathrm{Xe}^{*}\left({ }^{3} P_{1}\right), \mathrm{Xe}^{*}\left({ }^{3} P_{2}\right), \mathrm{Xe}_{2}^{*}\left(O_{u}^{+}\right)$, $\mathrm{Xe}_{2}^{*}\left({ }^{1} \Sigma_{u}^{+}\right), \mathrm{Xe}_{2}^{*}\left({ }^{3} \Sigma_{u}^{+}\right)$, and $\left.\mathrm{Xe}^{* *}\right) . \mathrm{Xe}^{* *}$ stands for the sum of $6 s^{\prime}, 6 p, 5 d, 7 s$ states. The terms $n_{i}, \boldsymbol{\Gamma}_{i}, S_{i}, D_{i}, \mu_{i}, \mathbf{E}$, $\epsilon$, and $q_{i}$ are the number density, flux, source function, diffusion coefficient, mobility, electric field, dielectric permittivity, and charge for species $i$, respectively; $q_{i}$ and $\operatorname{sgn}\left(q_{i}\right)$ for excited species are zero.

Equation (1) is the continuity equation for the density $n_{i}$ of species $i$. Equation (2) is the momentum transfer equation for the particle flux $\boldsymbol{\Gamma}_{i}$ in the drift-diffusion approximation. It includes the mobility-driven drift term and the diffusion term. Equation (3) is Poisson's equation for the electric field E.

The local field approximation ${ }^{3-7}$ is used for computing source function and transport coefficient: the source function $S_{i}$ in Eq. (1) and the mobility coefficient $\mu_{i}$ in Eq. (2) are assumed to be functions of the reduced electric field $E / P(E$ is electric field strength and $P$ is the neutral gas pressure). This is sufficient approximation for our purpose..$^{6}$ The relations between $S_{i}, \mu_{i}$, and $E / P$ are obtained using the Boltzmann solver, BOLSIG. ${ }^{3,14}$ The diffusion coefficient $D_{i}$ is obtained from the Einstein relation

$$
D_{i}=\frac{\mu_{i} k_{B} T_{i}}{e},
$$

where $k_{B}$ is the Boltzmann constant, $e$ is the elementary charge, and $T_{i}$ is the particle temperature. For the estimation of $D_{i}$, it is assumed that electron and ion temperatures are 1 $\mathrm{eV}$ and the gas temperature $350 \mathrm{~K}$, respectively.

At the interface between the gas and the dielectric surface, the charge density is calculated by integrating the charged particle fluxes to the surfaces during the discharge evolution. The particle fluxes to a dielectric surface are calculated, as in Ref. 3

$$
\begin{aligned}
& \boldsymbol{\Gamma}_{p} \cdot \mathbf{n}=\operatorname{sgn}\left(q_{p}\right) s_{p} \mu_{p} n_{p} \mathbf{E} \cdot \mathbf{n}+n_{p} \frac{\bar{v}_{p}}{4}, \\
& \boldsymbol{\Gamma}_{e} \cdot \mathbf{n}=-s_{e} \mu_{e} n_{e} \mathbf{E} \cdot \mathbf{n}+n_{e} \frac{\bar{v}_{e}}{4}+\boldsymbol{\Gamma}_{\mathrm{se}} \cdot \mathbf{n}, \\
& \boldsymbol{\Gamma}_{\mathrm{se}}=-\sum_{p} \gamma_{\mathrm{se}, p} \boldsymbol{\Gamma}_{p},
\end{aligned}
$$

where $e$ denotes electron and $p$ denotes ions or excited species. Equations (5) and (6) have the mobility-driven drift term and the isotropic thermal flux term. In addition, Eq. (6) for electron has an additional flux term $\boldsymbol{\Gamma}_{\text {se }}$ due to secondary electron emission by ion or metastable impact. The term $\gamma_{\mathrm{se}}$ in Eq. (7) is the secondary electron emission coefficient, $\mathbf{n}$ is the unit normal vector towards the wall, and $\bar{v}_{i}$ is mean speed of species $i$

$$
\bar{v}_{i}=\sqrt{\frac{8 k_{B} T_{i}}{\pi m_{i}}} .
$$

The symbol $s$ is defined in a way that it is nonzero only if the drift velocity is directed towards the wall

$$
s=\left\{\begin{array}{c}
1 \operatorname{sgn}(q) \mu \mathbf{E} \cdot \mathbf{n}>0 \\
0 \operatorname{sgn}(q) \mu \mathbf{E} \cdot \mathbf{n} \leqslant 0 .
\end{array}\right.
$$

We use the Neumann boundary condition for the open boundary, i.e., the gradients of densities and fluxes on the open boundary are zero.

The densities of the excited species decay as they radiate the UV or IR photons. The nonresonant IR or UV photons are assumed to reach the wall very rapidly without being trapped by the ground state neutral species. However, the resonant UV photons emitted from $\mathrm{Xe}^{*}\left({ }^{3} P_{1}\right)$ suffer many absorptions and reemissions before they reach the wall. The strict kinetics of the resonant photons is described by solving the Holstein equation ${ }^{15}$ or using a multiple-scattering representation, ${ }^{16,17}$ which is very time consuming. Therefore, the concept of an escape factor is often used for an approximate formulation of incorporating trapping effects, especially in PDP simulation. In our simulation the formula in Ref. 3 is used for calculating the escape factor.

Under the assumption of the isotropic emission of UV photons, their fluxes to the phosphor are computed using ray-tracing techniques. ${ }^{18}$ Some UV photons reaching the phosphor are converted into the visible photons according to a quantum efficiency of the phosphor for UV-visible photon conversion. Then we assume that the visible photons are isotropically reemitted and transparent to the gas. The fluxes of the visible photons arriving on the output window are again calculated using ray-tracing techniques. The obtained results are used for calculating the luminance and luminous efficiency.

\section{B. Definitions of physical quantities}

The formulas used for calculating the discharge and luminous efficiencies are as follows. The discharge efficiency (or UV radiation efficiency) is defined by ${ }^{3}$

$$
\eta_{\text {dis }}=\frac{W_{\mathrm{UV}}}{W_{\text {in }}},
$$

where $W_{\mathrm{UV}}$ and $W_{\text {in }}$ are the UV radiation energy and the Joule loss, respectively. The total energy of UV photons $W_{\mathrm{UV}}$ is

$$
W_{\mathrm{UV}}=\int_{\text {cycle }} d t \int_{\substack{\text { discharge } \\ \text { volume }}} d V \sum_{k} n_{k} \nu_{k} \epsilon_{k, h v},
$$

where $k, \nu$, and $\epsilon$ are excited species emitting UV photons, emission frequency, and energy of emitted photons, respectively. The Joule loss $W_{\text {in }}$ is

$$
W_{\text {in }}=\int_{\text {cycle }} d t \int_{\substack{\text { discharge } \\ \text { volume }}} d V \sum_{i} q_{i} \boldsymbol{\Gamma}_{i} \cdot \mathbf{E},
$$

where $i$ is charged species. The averaged photon power or consumed power is defined by

$$
P_{\mathrm{UV}, \text { in }}=\frac{W_{\mathrm{UV}, \text { in }}}{\int_{\text {cycle }}^{\text {half }} d t} .
$$


The luminous efficiency (or light generation efficiency) is defined by ${ }^{19}$

$$
\eta_{\text {vis }}=\frac{W_{\text {vis }}}{W_{\text {in }}},
$$

where $W_{\text {vis }}$ is the visible light energy. The total energy of visible photons $W_{\text {vis }}$ is

$$
W_{\text {vis }}=\int_{\text {cycle }} d t \int_{\text {window }} d S \Phi \epsilon_{\mathrm{ph}},
$$

where $\Phi$ and $\epsilon_{\mathrm{ph}}$ are visible photon flux and energy of visible photons, respectively. The luminance is given by

$$
L=\frac{W_{\text {vis }}}{\pi \int_{\substack{\text { half } \\ \text { cycle }}} d t \int_{\text {window }} d S} .
$$

While the photon power and discharge efficiency are not related to the position or area of the phosphor, the luminance and luminous efficiency are much dependent on it.

\section{Numerical method}

The coupled equations (1)-(7) are integrated between time $t^{k}$ and time $t^{k+1}=t^{k}+\Delta t$ where $\Delta t$ is a time step.

The continuity Eq. (1) is integrated by a Douglass and Gunn alternative direction implicit method ${ }^{20}$

$$
\begin{gathered}
\left(n_{i}-\frac{1}{2} \frac{\partial \Gamma_{i, x}}{\partial x} \Delta t\right)^{*}=\left(n_{i}+\left(\frac{1}{2} \frac{\partial \Gamma_{i, x}}{\partial x}+\frac{\partial \Gamma_{i, y}}{\partial y}+\frac{\partial \Gamma_{i, z}}{\partial z}\right.\right. \\
\left.\left.+S_{i}\right) \Delta t\right)^{k}, \\
\left(n_{i}-\frac{1}{2} \frac{\partial \Gamma_{i, y}}{\partial y} \Delta t\right)^{* *}=\left(n_{i}-\frac{1}{2} \frac{\partial \Gamma_{i, y}}{\partial y} \Delta t\right)^{*}, \\
\left(n_{i}-\frac{1}{2} \frac{\partial \Gamma_{i, z}}{\partial z} \Delta t\right)^{k+1}=\left(n_{i}-\frac{1}{2} \frac{\partial \Gamma_{i, z}}{\partial z} \Delta t\right)^{* *} .
\end{gathered}
$$

The superscript $k$ and the subscript $i$ represent the time index and the species index, respectively. The superscript asterisks and double asterisks denote intermediate values. Because this method is not explicit but implicit, it is stable and accurate for time steps much larger than the Courant-FriedrichsLewy (CFL) condition. ${ }^{21}$

For the calculation of the values $\Gamma_{i, x}^{*}, \Gamma_{i, y}^{*}, \Gamma_{i, y}^{* *}, \Gamma_{i, x}^{* *}$, and $\Gamma_{i, z}^{k+1}$, we need the values of $\mathbf{E}^{*}, \mathbf{E}^{* *}$, and $\mathbf{E}^{k+1}$, which are unknown in time $t^{k}$. When for these electric fields the value $\mathbf{E}^{k}$ is used in Eqs. (17)-(19), the simulation time step should not be larger than the dielectric relaxation time ${ }^{22}$

$$
t_{d}=\frac{\epsilon_{0}}{\sum_{i}\left|q_{i}\right| \mu_{i} n_{i}},
$$

where $t_{d}$ is inversely proportional to the plasma density $n$. When the discharge is ignited, the plasma density grows exponentially and the simulation time step should be reduced accordingly. This is the most crucial factor to slow down the calculation speed of the simulation code. To relax this kind of restriction on reducing the time step, we use the estimated electric field $\widetilde{\mathbf{E}}^{k+1}$ for the values $\mathbf{E}^{*}, \mathbf{E}^{* *}$, and $\mathbf{E}^{k+1}$.

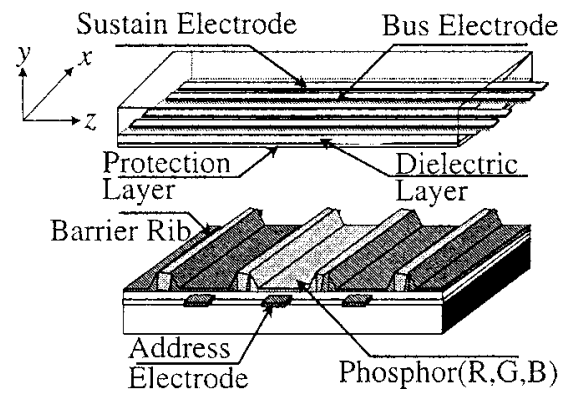

FIG. 1. Schematic diagram of a typical PDP cell.

We obtain the electric field $\widetilde{\mathbf{E}}^{k+1}$ from Poisson's equation

$$
\nabla \cdot\left(\epsilon \tilde{\mathbf{E}}^{k+1}\right)=\sum_{i} q_{i} \tilde{n}_{i}^{k+1}
$$

Note that instead of the actual density at time $t^{k+1}$, the estimated density $\tilde{n}_{i}^{k+1}$ is used in Eq. (21), where $\tilde{n}_{i}^{k+1}$ is calculated from

$$
\tilde{n}_{i}^{k+1}=n_{i}^{k}-\Delta t \nabla \cdot \Gamma_{i}\left(n_{i}^{k}, \tilde{\mathbf{E}}^{k+1}\right) .
$$

With the substitution of Eq. (2) into Eq. (22), Eqs. (21) and (22) become be $^{23}$

$$
\begin{gathered}
\nabla \cdot\left\{\left[\boldsymbol{\epsilon}+\sum_{i} \Delta t n_{i}^{k} \mu_{i}^{k} \operatorname{sgn}\left(q_{i}\right)\right] \widetilde{\mathbf{E}}^{k+1}\right\} \\
=\sum_{i}\left[q_{i} n_{i}^{k}+\Delta t \nabla \cdot\left(D_{i}^{k} \nabla n_{i}^{k}\right)\right] .
\end{gathered}
$$

The modified Poisson's Eq. (23) is discretized spatially according to the seven-point formula, and is solved by the successive over-relaxation algorithm with Chebyshev acceleration.

The flux in Eq. (2) is spatially discretized using the Sharfetter-Gummel scheme ${ }^{24}$ which supports large density gradients. Spatially discretized forms of Eqs. (17)-(19) are converted to tridiagonal systems of equations which can be easily solved.

As we mentioned it in Sec. I, implicit or semi-implicit algorithm makes it possible to make use of simulation time steps as large as 50 times of the CFL condition and 20 times of the dielectric relaxation time. It takes about one day on a Pentium PC to simulate five voltage pulses for a $64 \times 43 \times 12$ grid system, which is suitable to obtain steady state results at a relatively small computational time.

In this section, we described the physical and numerical model for our PDP simulation and the definitions of some important terms in this field. We also validated the reliability of our code through various comparisons though the details are not published: comparisons with the analytic Paschen theory for a parallel-plate geometry and diagnostics from other simulation codes ${ }^{11,12}$ for a coplanar PDP geometry.

\section{SIMULATION RESULTS IN THE SUSTAIN MODE}

Figure 1 shows the schematic diagram of a coplanar ac PDP. Two sustain electrodes (scan electrode and common electrode) and one address electrode make a discharge cell. 


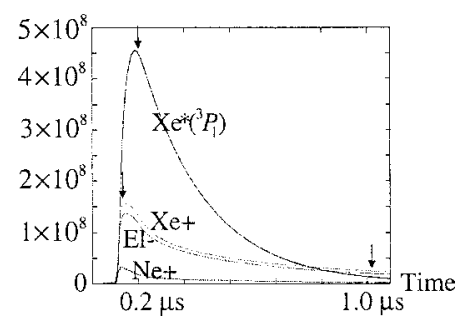

FIG. 2. Total number traces of xenon ion, neon ion, electron, and resonant excited xenon in the sustain mode.

In our simulation, the cell is turned on through two steps. The first step is the discharge ignition between scan and address electrodes, which is called addressing. The second step is the ac pulsed discharges between two sustain electrodes, which is called sustaining. The addressing is just a transient discharge to provide the selectivity of the cell. Most of the visible photons are generated during the sustain pulse. Therefore, the luminance or the efficiency of PDP is determined by the sustaining discharges. From the addressing to the steady pulsed discharges (sustaining), usually ten or more transient pulsed discharges exist. Therefore, we should simulate several pulses for meaningful analysis of the efficiency by simulation. This simulation is very time consuming, especially for three-dimensional systems. There are not many reports on the simulation of sustaining discharges of PDP in three dimensions. In this article, we present the discharge characteristics of the three-dimensional simulation in the sustain mode.

The simulation parameters are as follows. The cell sizes are shown in each figure. The distance between two sustain electrodes is $60 \mu \mathrm{m}$. The neutral gas is a neon-xenon (96/ 4\%) mixture with the pressure 500 Torr. The secondary electron emission coefficients on the protection layer are 0.5 , 0.05 , and 0.5 for neon ions, xenon ions, and xenon-excited species, respectively. A sustain voltage with a frequency of $100 \mathrm{kHz}$ and a duty ratio of $30 \%$ is applied.

Figure 2 shows the total number traces of electron, ions, and resonant excited xenon during the sustain pulse. At the times indicated by arrows in Fig. 2, various spatial profiles are calculated: top view and side view of the resonant excited xenon density [Figs. 3(a) $-3(\mathrm{c})$ and 3(d) $-3(\mathrm{f})$ ], and wall charge distributions on the upper dielectric surface and barrier rib [Figs. 4(a)-4(c) and 4(d)-4(f)]. In Fig. 3, the contours are equipotential lines and the shadings represent the density of resonant excited xenon. Top views and side views are taken from the plane where the density is the maximum in the $y$ direction at the number peak time and the plane where the $z$ position is the middle of the $z$ direction, respectively.

As time advances, the discharge occurs near the gap with the help of applied potential and wall charge generated from the previous discharge. The number of plasma particles increases rapidly and wall charges are accumulated on the dielectric surface. Then the plasma particles spread over the upper dielectric surface due to the accumulation of wall charge on the upper dielectric surface [Figs. 3(a)-3(c)]. The accumulation also starts at the center region and moves to

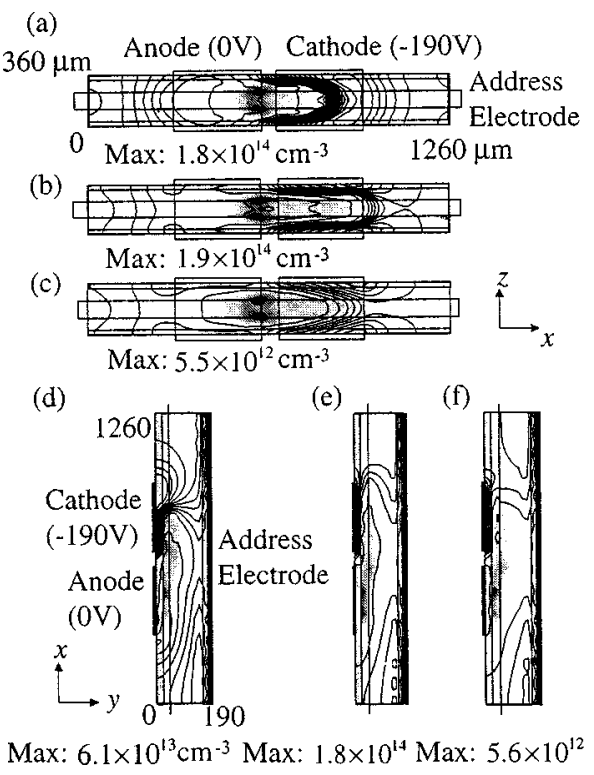

FIG. 3. (a)-(c) Top view and (d)-(f) side view of the contour plots of the resonant excited xenon density and potential at times denoted by arrows in Fig. 2 .

the outer side of the electrodes [Figs. 4(a)-4(c)]. Since the polarity of wall charges is opposite to the applied voltage, the electric field inside the cell is decreased, which leads to self-extinction of the discharge. Interestingly, the top view of the resonant excited xenon density shows the two bumps in the $z$ direction [Figs. 3(a)-3(c)] as in experimental result of Ref. 25. They are formed because of the cathode sheath near barrier ribs. The number of bumps depends on the geometry of the PDP cell. Figures 6(a)-6(c) show only one bump.

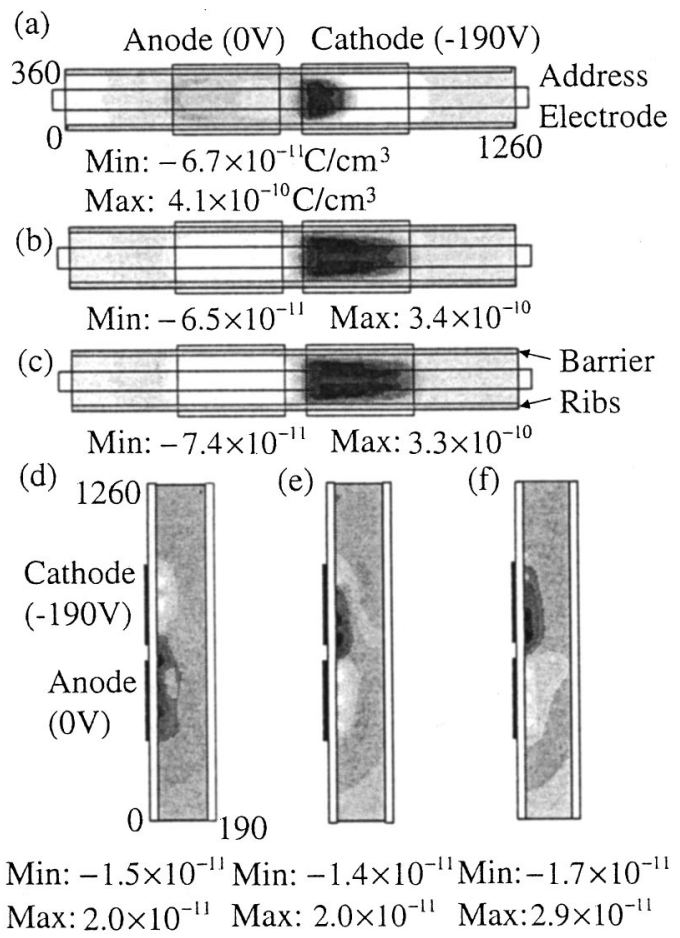

FIG. 4. Wall charge distributions on (a)-(c) the upper dielectric surface and (d)-(f) the barrier rib at times denoted by arrows in Fig. 2. 
In the following subsections, first we describe the threedimensional features of the wall charge distributions. Second, the local discharge efficiency is obtained and analyzed. Finally, we investigate the effects of the three-dimensional parameters, such as (i) electrode shape, (ii) barrier ribs, and (iii) width of address electrode, which cannot be included in usual two-dimensional simulations. As will be seen later, these simulations are helpful for suggesting new designs of PDP cell for its optimization.

\section{A. Wall charge distribution}

The wall charge affects the discharge so much and also plays a very important role on the operation of ac PDP. During the discharge, because the charges accumulated on the dielectric surfaces reduce the effect of the applied voltage, the discharge is self-extinguished. Since the sustain voltage itself is smaller than the firing voltage, the discharge cannot be ignited by itself without the help of the wall charge accumulated by the previous discharge.

It is hard to directly observe the wall charge distribution in experiment, but it can be easily obtained in simulation. The wall charge can be accumulated on the upper and lower dielectric surfaces and barrier ribs. However, since the wall charge on the lower dielectric surface does not usually play an important role on the discharge in the sustain mode, we diagnose the wall charges only on the upper dielectric surface and barrier ribs. Here we present the three-dimensional features of the wall charge distributions: the $z$-directional distribution of the wall charge on the upper dielectric layer and the distribution on barrier ribs that cannot be included in the two-dimensional simulation.

In side views of the resonant excited xenon profiles [Figs. 3(d) $-3(\mathrm{f})]$, the striations are observed. The experimental and simulational observations of striations in PDP have been reported elsewhere. ${ }^{26-29}$ Noticeably, wall charge distribution on the barrier rib shows the striations near the cathode as well as the anode [Figs. 4(d)-4(f)], though the striations of plasma particles are formed only in the anode side [Figs. $3(d)-3(f)] .{ }^{26-29}$ The striations of wall charge near the cathode come from the previous discharge when the present cathode was actually an anode.

In wall charge distributions on the upper dielectric surface [Figs. 4(a)-4(c)], there is nonuniformity along the $z$ direction and the positive wall charge on the cathode side is accumulated more nonuniformly than the negative wall charge on the anode side (though it is hard to resolve the uniformity well in figures). We can also ensure it through the comparison of wall charge density on the barrier ribs [Figs. 4(d) -4(f)] and on the upper dielectric surface [Figs. 4(a)4(c)]. On the anode side, wall charge on the barrier rib is five times smaller than that of the upper dielectric surface and on the cathode side, over ten times. Thus there is the significant nonuniformity along the $z$ direction and the nonuniformity is higher near the cathode than near the anode. This is one of the three-dimensional results from the widely known properties that the mobility of electrons is higher than that of ions. This result means that the two-dimensional simulation overestimates the uniformity of wall charge, especially positive wall charge, because the $z$ direction is assumed to be infinite in that simulation.

\section{B. Local discharge efficiency}

In general, the discharge efficiency is increased by decreasing the electron temperature or the electric field. ${ }^{3}$ As the ratio of the discharge in the small electric field region to the discharge in the large electric field region increases, the efficiency increases. We can adapt this result to a PDP cell. In a coplanar PDP cell, there is the short gap between two sustain coplanar electrodes where the large electric field exists and, consequently, the discharge initiates. Thus, in order to increase the efficiency of a cell, we should design a new cell in which the discharge is activated at the outer region of electrodes where the electric field is low. When the discharge at the outer region is more activated, the discharge will form wider plasma distribution along the dielectric surface, which is called the long path discharge. This can be one of the reasons why a longer path discharge leads to higher efficiency.

However, in an ac PDP cell, since the surface charge affects the discharge so much, as mentioned in Sec. III A, the above statements cannot be true. The charge accumulation on the dielectric surface reduces the effect of the applied potential and the electric field structure is greatly changed. We investigate it through the local efficiency plot and suggest how to design a cell with the high efficiency.

The efficiency in PDP terminology is the averaged quantity in time and space as defined in Eq. (10). Here, in order to analyze the details of efficiency, we define a new local discharge efficiency as the ratio of photon power to consumed power at each position. That is, the local efficiency is the time-averaged but space-resolved quantity. But a detailed discussion about this definition is needed. The meaningful definition of the local efficiency is how much ratio of total consumed power is deposited to the photon production at each position. However, we may have difficulty in calculating it. The positions of the deposited power and the photon production could be different since the excited species created from the consumed power in some positions is transported to another position before the photon is emitted from it. But because the transport of the excited species is driven by the diffusion motion due to the density gradients rather than the drift motion due to the electric field, it is assumed that there is no big difference at least in the spatial distribution of the local efficiency.

Figures 5(a)-5(c) are the averaged photon power, power consumption, and local discharge efficiency. They are averaged over a half cycle in time and $y$ direction in space. The region where the efficiency is high or low is distinguished mainly by the cathode or the anode side rather than by the inner edge or the outer edge of the electrodes. This comes from the charge accumulation on the surface as stated. Nearly the same number of photons is generated near the two electrodes, while power consumption is larger near the cathode than near the anode. Hence, the local discharge efficiency is higher near the anode than near the cathode. That is because in the cathode side there is the cathode sheath region 


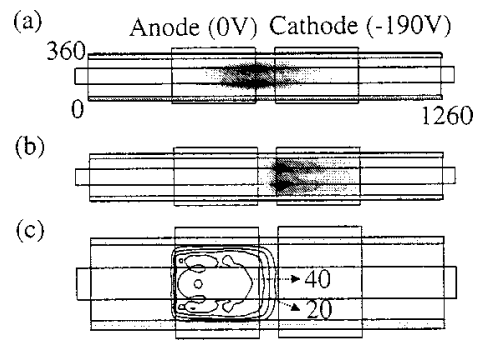

FIG. 5. Time-averaged (a) photon power, (b) power consumption, and (c) local discharge efficiency. They are spatially averaged in the $y$ direction.

where the electric field is large and consequently the production of xenon excited states is low, ${ }^{3}$ while in the anode side there is the plasma column region where the electric field is small and, consequently, the xenon excited states are produced efficiently. Therefore, for the high efficiency we should design a new PDP cell where the discharge is activated more in the plasma column region than in the cathode sheath region. From this point of view, the long path discharge with the long plasma column region can be included in the category. In addition, we also notice that the discharge efficiency is higher near the center of the anode than near the edge of the anode and barrier ribs.

\section{Effect of electrode shaping}

The discharge characteristics of the PDP cell are much dependent on the shape of sustain electrode. Though the electrode shaping needs more efforts to make it, it can be used for the further optimization of PDP cell. One of those examples is a T-shaped electrode cell with about $15 \%$ higher efficiency than that of a conventional electrode cell. ${ }^{8}$ Through our three-dimensional simulations, we investigate why a T-shaped electrode cell shows the higher efficiency and why power consumptions are nearly the same, although the area of T-shaped electrode is smaller than that of conventional one.

Figures 6(a)-6(c) and 6(d)-6(f) are top views of the resonant excited xenon density of a conventional electrode cell and T-shaped electrode cell, respectively. In both cases, the distance between scan and common electrodes is set to $65 \mu \mathrm{m}$. At the number peak the maximum density is smaller in the T-shaped one than in the conventional one, but the total number is the larger in the T-shaped one. Since a T-shaped electrode is longer along the $x$ direction than a conventional electrode, the plasma distribution in a T-shaped electrode cell is wider along the $x$ direction than that in the conventional one [Figs. 6(a)-6(c) and 6(d)-6(f)]. The T-shaped electrode cell has a longer plasma column region than a conventional one. This is why the discharge efficiency of T-shaped electrode is about 20\% higher than that of a conventional one as shown in Fig. 7(a). Therefore, the efficiency improvement of the T-shaped electrode is one of the examples included in the statement in Sec. III B.

In the case of a conventional electrode cell, it is widely known that as its electrode is wider along the $x$ direction the plasma distribution is wider and power consumption increases. We investigate that the electrode area is not always

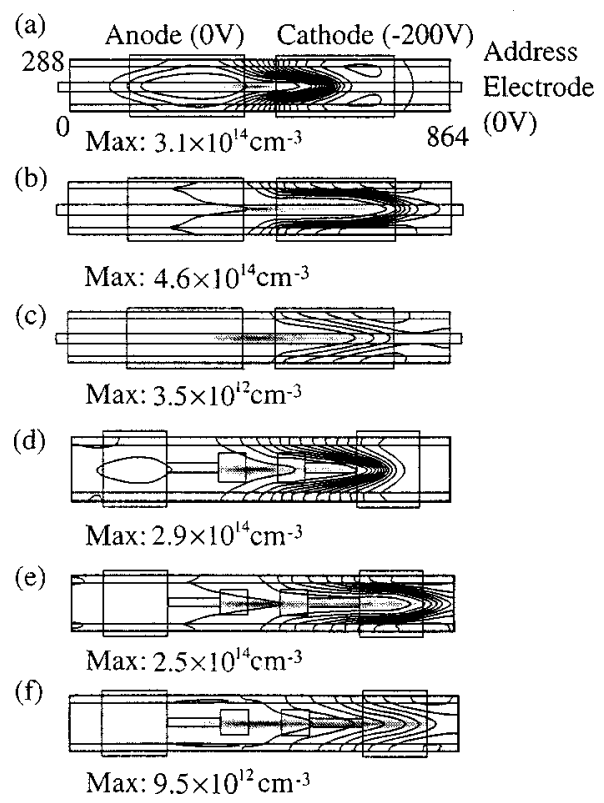

FIG. 6. Top view of the contour plots of the resonant excited xenon density and potential in (a) a conventional electrode cell and (b) T-shaped electrode cell.

proportional to power consumption. The area of the T-shaped electrode is just $77 \%$ of that of the conventional electrode, but its power consumption is comparable with that of the conventional one as shown in Fig. 7(b). The explanation about it is as follows. Since the discharge in this simulation is confined to the center region in the $z$ direction [Figs. 6(a)6(c) and 6(d)-6(f)], the electrode near the barrier ribs does not contribute so much to the discharge. The T-shaped electrode is thinner along the $z$ direction and longer along the $x$ direction than the conventional electrode. Thus, a larger part of T-shaped electrode than that of conventional one contributes to the discharge and power consumption. It means that,
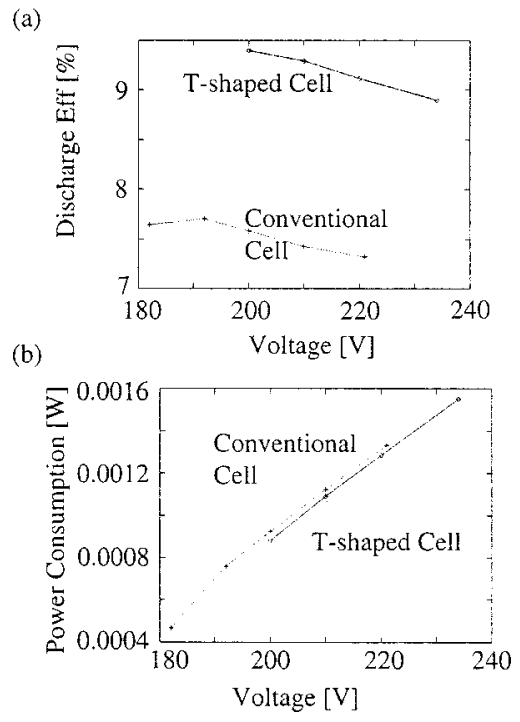

FIG. 7. Comparison between the conventional cell and T-shaped electrode cell. (a) Discharge efficiency vs sustain voltage. (b) Power consumption vs sustain voltage. 


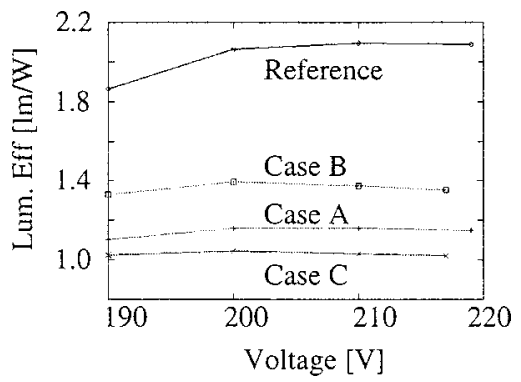

FIG. 8. Luminous efficiency vs sustain voltage for four cells, i.e., Reference, Case A, Case B, and Case C.

even if the efficiencies of two cells are the same, enough luminance with a relatively smaller area of electrodes in the T-shaped electrode can be achieved.

\section{Effect of barrier ribs}

Barrier ribs are used to prevent the electrical and optical interaction between cells. Since barrier ribs are coated with phosphors, they also contribute to the UV-visible photon conversion. We investigate the effects of barrier ribs on the efficiency.

In Sec. III B, we found out that the discharge efficiency near the edge of barrier ribs is rather low through Fig. 5(c). In this subsection, in order to find out how much barrier ribs influence on the overall efficiency, the following four cells are simulated-Reference, Case A, Case B, and Case C. "Reference" is the usual cell with barrier ribs that the phosphor is deposited on. Its cell size is $1140 \times 180 \times 380 \mu \mathrm{m}^{3}$. "Case A" has barrier ribs without the phosphor deposition. "Case B" and "Case C" do not have even barrier ribs. The cell size of Case B is the same as Reference, but in Case C the discharge volume instead of its cell size is the same as Reference. That is, the cell sizes of Reference, Case A, and Case B are the same, while that of Case C is $1140 \times 180 \times 300$ $\mu \mathrm{m}^{3}$.

Figure 8 is the luminous efficiencies of four cells. Case B has around $44 \%$ lower efficiency than Reference. In our simulation, the contribution of barrier ribs is underestimated because the barrier rib is described as a rectangular parallelepiped in simulation unlike a real PDP cell. We can, therefore, notice that the phosphor on barrier ribs contributes to over $44 \%$ of total luminance. The remaining $56 \%$ comes from the bottom phosphor on the lower dielectric. The phosphor area on barrier ribs is about $45 \%$ of the overall phosphor area, which is nearly the same as the percentage of its contribution to efficiency. This relation makes it easy to estimate the contribution of some phosphor region in arbitrary geometry.

In comparisons between Case A and Case B or Case A and Case $\mathrm{C}$, there is no significant difference. This result gives the conclusion that barrier ribs themselves do not play an important role in the overall discharge efficiency. This agrees well with the result that there are not many particles near barrier ribs [Figs. 6(a) $-6(\mathrm{c})$ and 6(d) $-6(\mathrm{f})]$.

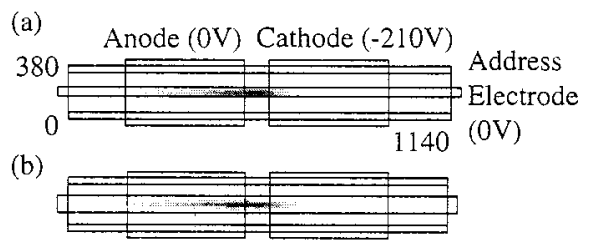

(c)

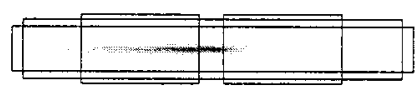

(d)

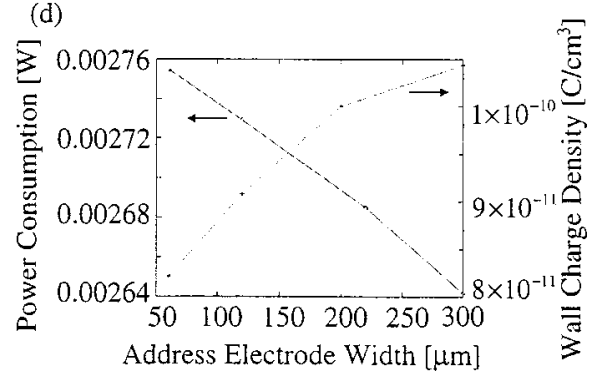

FIG. 9. Top views of the contour plots of the electron density for the various values of address electrode width; i.e., (a) $60 \mu \mathrm{m}$, (b) $120 \mu \mathrm{m}$, and (c) 300 $\mu \mathrm{m}$. (Contours are plotted at each number peak time with the same contour level values.) (d) Power consumption and wall charge density vs address electrode width. (Wall charge density is taken as the difference between maximum and minimum values.)

\section{E. Effect of address electrode width}

Address electrode is used to select the cell that should be turned on or off. It also defines the position of the discharge in the direction perpendicular to the sustain electrodes. Here we investigate how much the width of address electrode affects the size of the discharge in the sustain mode when the address electrode is grounded.

Figures 9(a)-9(c) are electron densities at each number peak time for various values of address electrode width, i.e., 60,120 , and $300 \mu \mathrm{m}$, respectively. The size of the discharge in the $z$ direction is nearly independent of the address electrode width. As address electrode width increases, power consumption is slightly reduced but wall charge density on barrier ribs increases as shown in Fig. 9(d). Here wall charge density is taken as the difference between maximum and minimum values. It means that, though the large electric field region is expanded, the number of the plasma particles does not increase because of the wall loss. The particle loss to barrier ribs and its charging prevent the expansion of the discharge. This is why the address electrode width does not affect the size of the discharge in the $z$ direction in our simulation even though too small width of the address electrode makes the addressing impossible. It may not be general and limited only to our condition. Nonetheless, it is found that the address electrode width is not always proportional to the size of the discharge because of the wall loss of the particles to barrier ribs.

\section{CONCLUSIONS}

Three-dimensional simulation is useful for understanding of the three-dimensional features and further optimization of ac PDP. For this purpose, we have developed a threedimensional fluid code (FL3P) with the remarkable 
improvement of computation speed by employing semiimplicit method. Using this simulator, we obtain various results about the three-dimensional features in the sustain mode, which are directly related to the efficiency.

The three-dimensional features of discharges were investigated in the sustain mode of PDP. First, we observed the striations of wall charge at both the anode and cathode side unlike the striations of plasma particles for the first time. We also found that the two-dimensional simulation overestimates the uniformity of wall charge, especially positive wall charge. Second, the local discharge efficiency was obtained as a function of position in the $x z$ plane. It was mainly divided into the anode region and the cathode region and highest near the anode center. From this result, we suggest that the plasma column should be enhanced for the improvement of efficiency. Finally, we study the effects of the threedimensional parameters such as electrode shape, barrier ribs, and width of address electrode, which cannot be included in usual two-dimensional simulations. As one of the examples showing the effect of electrode shaping, we compare the discharge characteristics of a T-shaped electrode cell with that of a conventional cell. From these comparisons, we found that the high efficiency of the T-shaped electrode cell can be explained by the long plasma column and the T-shaped electrode can be used for producing enough luminance with a relatively small area of electrodes. We also obtain the results that the phosphor on barrier ribs contributes to over $44 \%$ of total luminance, but barrier ribs themselves do not play an important role in the overall discharge efficiency. Address electrode width was not always proportional to the size of the discharge in the $z$ direction because of the wall loss of the particles to barrier ribs. In the future, these results through the three-dimensional simulation will give us more extensive and helpful knowledge to optimize the PDP cell.

\section{ACKNOWLEDGMENT}

The financial support from LG Electronics and the Ministry of Education of Korea through its BK21 program is gratefully acknowledged.
${ }^{1}$ A. Sobel, IEEE Trans. Plasma Sci. 19, 1032 (1991).

${ }^{2}$ O. Sahni, C. Lanza, and W. E. Howard, J. Appl. Phys. 49, 2365 (1978).

${ }^{3}$ J. Meunier, P. Belenguer, and J. P. Boeuf, J. Appl. Phys. 78, 731 (1995)

${ }^{4}$ R. Veerasingam, R. B. Campbell, and R. T. McGrath, Plasma Sources Sci. Technol. 6, 157 (1997).

${ }^{5}$ C. Punset, J. P. Boeuf, and L. C. Pitchford, J. Appl. Phys. 83, 1884 (1998).

${ }^{6}$ P. J. Drallos, V. P. Nargony, and W. Williamson, Plasma Sources Sci. Technol. 4, 576 (1995).

${ }^{7}$ R. B. Campbell, R. Veerasingam, and R. T. McGrath, IEEE Trans. Plasma Sci. 23, 399 (1995)

${ }^{8} \mathrm{~K}$. Amemiya, T. Komaki, and T. Nishio, Proceedings of the Fifth International Display Workshops (IDW'98), Kobe, Japan, 1998, p. 531

${ }^{9}$ O. Toyoda, T. Kosaka, F. Namiki, A. Tokai, H. Inoue, and K. Betsui, Proceedings of the Sixth International Display Workshops (IDW'99), Sendai, Japan, 1999, p. 599

${ }^{10}$ C. K. Yoon, J. H. Yang, W. J. Chung, K. C. Choi, and K. W. Whang, Proceedings of the Seventh International Display Workshops (IDW'00), Kobe, Japan, 2000, p. 627.

${ }^{11}$ H. S. Jeong, Y. Murakami, and M. Seki, Proceedings of the Sixth International Display Workshops (IDW'99), Sendai, Japan, 1999, p. 671.

${ }^{12}$ Y. K. Shin, C. H. Shon, W. Kim, and J. K. Lee, IEEE Trans. Plasma Sci. 27, 1366 (1999).

${ }^{13}$ M. S. Hur, J. K. Lee, C. H. Shon, and S. S. Yang, Proceedings of the Seventh International Display Workshops (IDW'00), Kobe, Japan, 2000, p. 715.

${ }^{14}$ The Siglo Database, CPAT and Kinema Software, http://www.kinema.net/ siglo, 1998.

${ }^{15}$ T. Holstein, Phys. Rev. 72, 1212 (1947); 83, 1159 (1951).

${ }^{16}$ J. B. Anderson, J. Maya, M. W. Grossman, R. Lagushenko, and J. F. Waymouth, Phys. Rev. A 31, 2968 (1985).

${ }^{17}$ A. F. Molisch, B. P. Oehry, W. Schupita, and G. Magerl, Comput. Phys. Commun. 93, 127 (1993)

${ }^{18}$ S. Rauf and M. J. Kushner, J. Appl. Phys. 85, 3460 (1999).

${ }^{19}$ S. Rauf and M. J. Kushner, J. Appl. Phys. 85, 3470 (1999).

${ }^{20}$ J. Douglas and J. E. Gunn, Numer. Math. 6, 428 (1964).

${ }^{21}$ P. J. Roache, Computational fluid dynamics (Hermosa, Albuquerque, NM, 1976).

${ }^{22}$ M. S. Barnes, T. J. Colter, and M. E. Elta, J. Appl. Phys. 61, 81 (1987).

${ }^{23}$ P. L. G. Ventzek, R. J. Hoekstra, and M. J. Kushner, J. Vac. Sci. Technol. B 12, 461 (1994).

${ }^{24}$ D. L. Sharfetter and H. K. Gummel, IEEE Trans. Electron Devices 16, 64 (1967).

${ }^{25}$ K. Tachibana and S. Feng, J. Appl. Phys. 88, 4967 (2000).

${ }^{26} \mathrm{H}$. Uchiike, Proceedings of the 18th International Display Research Conference (Asia Display '98), Seoul, Korea, 1998, p. 195.

${ }^{27}$ G. S. Cho, E. H. Choi, Y. G. Kim, D. I. Kim, H. S. Uhm, Y. D. Joo, J. G. Han, M. C. Kim, and J. D. Kim, J. Appl. Phys. 87, 4113 (2000).

${ }^{28}$ T. Yoshioka, A. Okigawa, L. Tessier, and K. Toki, Proceedings of the Sixth International Display Workshops (IDW'99), Sendai, Japan, 1999, p. 603.

${ }^{29}$ C. H. Shon and J. K. Lee, Phys. Plasmas 8, 1070 (2001). 